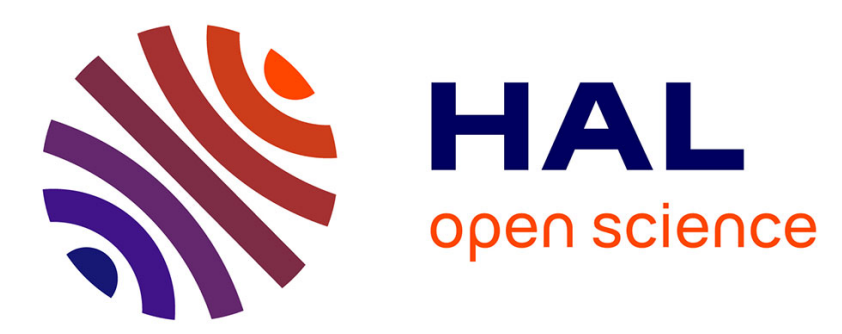

\title{
A method for the acquisition and the treatment of specific heat data-nuclear specific heat of $\mathrm{Cu}-\mathrm{Mn}$
}

P. Costa-Ribeiro, B. Picot, J. Souletie, D. Thoulouze

\section{To cite this version:}

P. Costa-Ribeiro, B. Picot, J. Souletie, D. Thoulouze. A method for the acquisition and the treatment of specific heat data-nuclear specific heat of $\mathrm{Cu}-\mathrm{Mn}$. Revue de Physique Appliquée, 1974, 9 (4), pp.749-756. 10.1051/rphysap:0197400904074900 . jpa-00243842

\section{HAL Id: jpa-00243842 https://hal.science/jpa-00243842}

Submitted on 1 Jan 1974

HAL is a multi-disciplinary open access archive for the deposit and dissemination of scientific research documents, whether they are published or not. The documents may come from teaching and research institutions in France or abroad, or from public or private research centers.
L'archive ouverte pluridisciplinaire HAL, est destinée au dépôt et à la diffusion de documents scientifiques de niveau recherche, publiés ou non, émanant des établissements d'enseignement et de recherche français ou étrangers, des laboratoires publics ou privés. 


\author{
Classification \\ Physics Abstracts \\ $0.624-7.540-8.533$
}

\title{
A METHOD FOR THE ACQUISITION AND THE TREATMENT \\ OF SPECIFIC HEAT DATA-NUCLEAR SPECIFIC HEAT OF Cu-Mn
}

\author{
P. COSTA-RIBEIRO $(*)$, B. PICOT, J. SOULETIE and D. THOULOUZE
}

Centre de Recherches sur les Très Basses Températures, C. N. R.S., B. P. 166, Centre de Tri, 38042 Grenoble-Cedex, France

(Reçu le 5 novembre 1973, révisé le 15 février 1974)

\begin{abstract}
Résumé. - Nous présentons une méthode de traitement des résultats de chaleur spécifique applicable aux très basses températures où les temps de réponses introduiraient de grandes erreurs dans la méthode graphique habituellement utilisée avec la technique adiabatique. Cette méthode d'acquisition et de traitement des données a été automatisée. La précision acquise nous permet de séparer les contributions respectives de l'impureté et de la matrice dans la chaleur spécifique nucléaire d'un alliage CuMn entre $20 \mathrm{mK}$ et $100 \mathrm{mK}$. On trouve $290 \pm 10 \mathrm{kOe}$ comme valeur du champ hyperfin sur les noyaux de Mn.
\end{abstract}

\begin{abstract}
A method is presented for the treatment of specific heat data at very low temperatures where long response times would introduce large errors in the standard graphical method used with the adiabatic technique : use is made of the knowledge of the time evolution of the temperature $T$ and of its time derivative $\dot{T}$. This method was adapted to an automatic system of acquisition and treatment of the data. The gain in accuracy enables us to separate the impurity and the host contribution to the nuclear specific heat of $\mathrm{Cu}-\mathrm{Mn}$ between $20 \mathrm{mK}$ and $100 \mathrm{mK}$. The hyperfine field is found to be $290 \pm 10 \mathrm{kOe}$ on the Mn nuclei.
\end{abstract}

1. Introduction. - We present a system of acquisition and treatment of data which was developed for measurements of specific heats in the range from $20 \mathrm{mK}$ to $100 \mathrm{mK}$, and was used in the study of the nuclear specific heat of dilute alloys (Pt-Co [1], Au-Co-Fe [2] and $\mathrm{Cu}-\mathrm{Mn}$ ).

The apparatus described in a former paper [3] is capable of cooling a sample down to about $15 \mathrm{mK}$ by the successive demagnetization of two salt pills. The sample is then isolated by decreasing the field on a superconducting thermal switch (a tin wire : $l=2 \mathrm{~cm}$ and $\varnothing=0.5 \mathrm{~mm}$ ). The heat leak $\dot{q}$ to the sample has been made as small as $1 \mathrm{erg} / \mathrm{min}$ by taking special care in protecting the apparatus from external perturbations (vibrations, electromagnetic radiations, etc...). The temperature is given by the magnetic susceptibility of a small sphere constituted of $5 \mathrm{CMN}$ slabs glued to the sample holder with Apiezon $N$ grease.

(*) In partial fulfilment of a thesis. C. A. P. E. S. Fellow (Brazil). Now in P. U. C. : Rua Marques de Sâo Vicente 209/263, Z C 20 Rio de Janeiro, Brésil.
2. The adiabatic method; difficulties inherent to the low temperature range. - A graph of the temperature of the sample versus time, typical of a specific heat experiment, is schematized (Fig. 1) in a somewhat ideal case : large diffusivity of the sample and instantaneous response of the thermometers. An additional

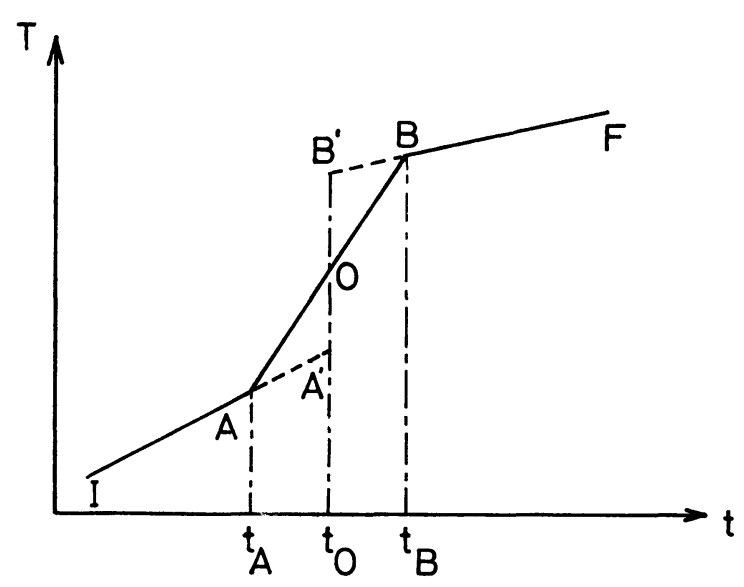

FIG. 1. - Schematized graphic of the temperature versus time, typical of a specific heat determination, in the simple case when the diffusivity is large and a fast response thermometer is used. 
controlled power $\dot{Q}$ is superimposed from $t_{\mathrm{A}}$ to $t_{\mathrm{B}}$ to $\dot{q}$, the heat leak to the sample, which is responsible for the drifts observed before $t_{\mathrm{A}}$ and after $t_{\mathrm{B}}$. The specific heat at $t_{0}$ is $C_{\mathrm{p}}=\Delta Q / \Delta T$, where $\Delta T=T_{\mathrm{B}^{\prime}}-T_{\mathrm{A}^{\prime}}$. $t_{0}$ is chosen in such a way that the areas of the triangles $\mathrm{OB}^{\prime} \mathrm{B}$ and $\mathrm{OA}^{\prime} \mathrm{A}$ are equal [4]. With a fast response time, the temperature change corresponding to $\dot{q}$ remains small between $t_{\mathrm{A}}$ and $t_{\mathrm{B}}$. Then the specific heat and the heat leaks may be considered constant and the drifts are approximately linear functions of time. In that case the extrapolation is easily made graphically. Unfortunately such is not the situation in the specific heat measurements at very low temperature, when thermal contact resistances exist between sample, sample holder and thermometer. With a CMN as the thermometer, time constants as large as $6 \mathrm{~min}$ were found at $20 \mathrm{mK}$. In figure 2 a mutual inductance bridge reading, typical of a low temperature measure-

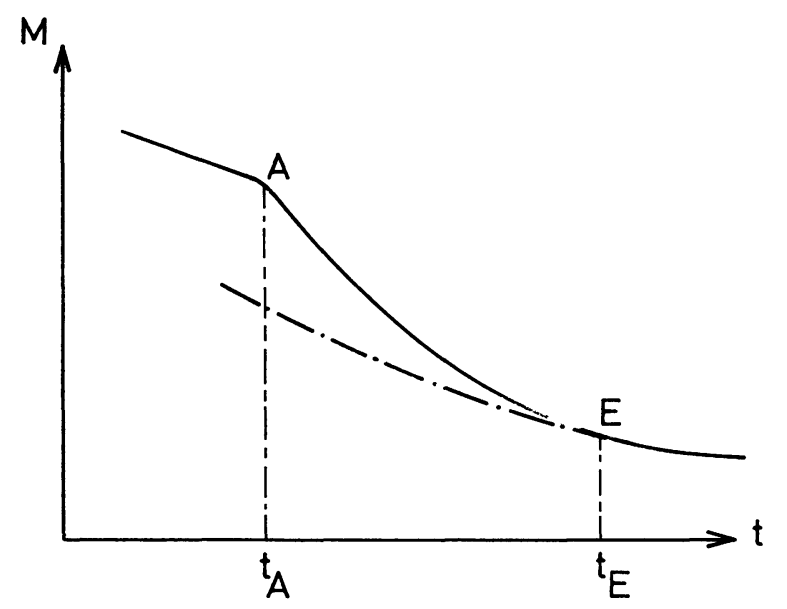

FIG. 2. - Evolution of the inductance bridge signal $M$ $(M=A / T+b)$ versus time when a heat pulse is applied to the sample at $t_{\mathrm{A}}$. After a transient period, dynamic equilibrium is recovered at $t_{\mathrm{E}}$. The dotted line shows the extrapolation backward in time made from the drifts after $t_{\mathrm{E}}$.

ment $(20 \mathrm{mK}$ to $70 \mathrm{mK}$ ), is plotted versus time $\left(M=b+\left(a / T^{\prime}\right), a\right.$ and $b$ being determined from a calibration in the helium range). Some typical difficulties arise then:

- From $t_{\mathrm{A}}$ to $t_{\mathrm{E}}$, the instant where the internal thermal equilibrium is recovered, the temperature of the thermometer will differ from that of the sample : this will make almost inoperable the application of the area rule in the determination of the instant $t_{0}$ where $T$ should be measured [4].

- On this time scale the temperature change produced by the natural heat leak $\dot{q}$ is comparable to or even larger than the externally supplied $\Delta Q . C_{\mathrm{p}}$ and $\dot{q}$ are no longer constant and the temperature drifts are non linear. But even if $C_{\mathrm{p}}$ and $\dot{q}$ remain reasonably constant over a large period of time, the $1 / T$ law followed by the thermometer will lead to non linear drifts of the inductance bridge signal. This makes a graphical extrapolation rather difficult.

- The time $t_{\mathrm{E}}$ from which this extrapolation is made is not easily determined on such a graphic as in figure 2.

In the following we will address ourselves to the last two problems, determination of $t_{\mathrm{E}}$ and extrapolation of the drifts, and will estimate the errors attached to the choice of $t_{0}$. The method proposed below for low temperature calorimetry with a CMN may be useful as well when faster thermometers are used, but other difficulties inherent to the sample are present, such as a large $T_{1}$ (for nuclear specific heat) or a bad diffusivity (for insulating systems).

3. The determination of $t_{E}$ - Curve A of figure $3 a$ schematizes the evolution of the temperature versus time due to $\dot{q}$ only ( free run »). $\dot{q}$ depends on $T$, the temperature of the sample, and on the temperature $T_{\mathrm{s}}$ of the screens with which the sample may exchange heat, by residual gas conduction, solid conduction and so on, and from its definition,

$$
\dot{q}\left(T, T_{\mathrm{s}}\right)=C_{\mathrm{p}}(T) \frac{\mathrm{d} T}{\mathrm{~d} t} .
$$

All the screens which surround the sample have been thermally anchored to the salt and $T_{\mathrm{s}}$ is observed to remain reasonably constant over a run. Then $\mathrm{d} T / \mathrm{d} t$ depends on $T$ only and the curve $\frac{\mathrm{d} T}{\mathrm{~d} t}(T)$ is a unique curve for a state of "dynamic equilibrium » characterized by the leaks $\dot{q}\left(T, T_{\mathrm{s}}\right)$ to the sample ; the curve $\mathrm{A}^{\prime}$ of figure $3 b$ schematizes the evolution of $\mathrm{d} T / \mathrm{d} t$ versus the temperature, corresponding to the path $\mathrm{A}$ of figure $3 a$.

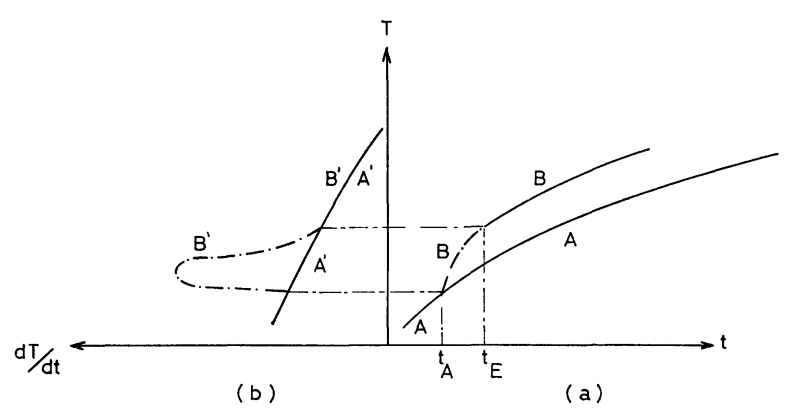

Fig. 3. - The evolution of the temperature $T$ versus time $t$ (Fig. a) and the corresponding time derivative of $T$ versus $T$ (Fig. b), for two cases : free run (curves $\mathbf{A}$ and $\mathrm{A}^{\prime}$ ) and specific heat measurement (curve $\mathrm{B}$ and $\mathrm{B}^{\prime}$ ). Before $t_{\mathrm{A}}$ (begining of the heat pulse) and after $t_{\mathrm{E}}$ (recovery of dynamic equilibrium) curves $\mathbf{A}^{\prime}$ and $\mathbf{B}^{\prime}$ coincide.

Now, when at $t_{\mathrm{A}}$ a heat pulse is applied to the sample, paths $\mathbf{B}$ (Fig. $3 a$ ) and $\mathbf{B}^{\prime}$ (Fig. $3 b$ ) will be described. However, after a transient period necessary for the total quantity of heat introduced to diffuse in the sample and in the thermometer, the 
system will come back at time $t_{\mathrm{E}}$ to the same dynamic equilibrium as before, characterized by $\dot{q}(T)$ and $\frac{\mathrm{d} T}{\mathrm{~d} t}(T)$. On figure $3 b$ the curves $\mathrm{A}^{\prime}$ and $\mathrm{B}^{\prime}$ coincide before $t_{\mathrm{A}}$ and after $t_{\mathrm{E}}$; over a complete run, discrete parts of $A^{\prime}$ are obtained and the total curve may be easily recovered by smooth interpolations between these parts. $t_{\mathrm{E}}$ is the instant at which $\mathbf{B}^{\prime}$ rejoins this interpolated curve of "dynamic equilibrium ".

The same considerations are also valid for $M$ and $\frac{\mathrm{d} M}{\mathrm{~d} t}(M)$ to which we have a direct access. Moreover, $\mathrm{d} M / \mathrm{d} t$ is a more physical quantity since, as shown in references [5] and [6], it is proportional to the heat leaks to the sample if the specific heat is proportional to $T^{-2}$ (as is generally the case at low temperature) :

$$
\dot{q}=\frac{A}{T^{2}} \frac{\mathrm{d} T}{\mathrm{~d} t}=-A \frac{\mathrm{d}}{\mathrm{d} t}\left(\frac{1}{T}\right)=-\frac{A}{a} \frac{\mathrm{d} M}{\mathrm{~d} t} .
$$

In practice, simultaneously with $M$ versus time, we record, on an XY recorder, $\frac{\mathrm{d} M}{\mathrm{~d} t}(M)$ which happens to be very linear over a complete run from 20 to $100 \mathrm{mK}$. This makes the determination of $t_{\mathrm{E}}$ particularly easy during the experiment. On figure $4 a$ typical XY plot of $\mathrm{d} M / \mathrm{d} t$ versus $M$ is shown over two runs. The return to dynamic equilibrium is followed over several heat pulses. The obvious advantage of working with $\frac{\mathrm{d} M}{\mathrm{~d} t}(T)$ rather than with $M(t)$ in the determination of $t_{\mathrm{E}}$ relies on the fact that the discontinuities, even when smoothed by large time constant effects, remain more apparent on the time derivative.

4. The extrapolation of the drifts. - The knowledge of $\mathrm{d} M / \mathrm{d} t$ makes possible the extrapolation of $M(t)$. This was the basis of a graphical method proposed in reference [6]. In our case, the empirical law

$$
\frac{\mathrm{d} M}{\mathrm{~d} t}=-\alpha M+\beta
$$

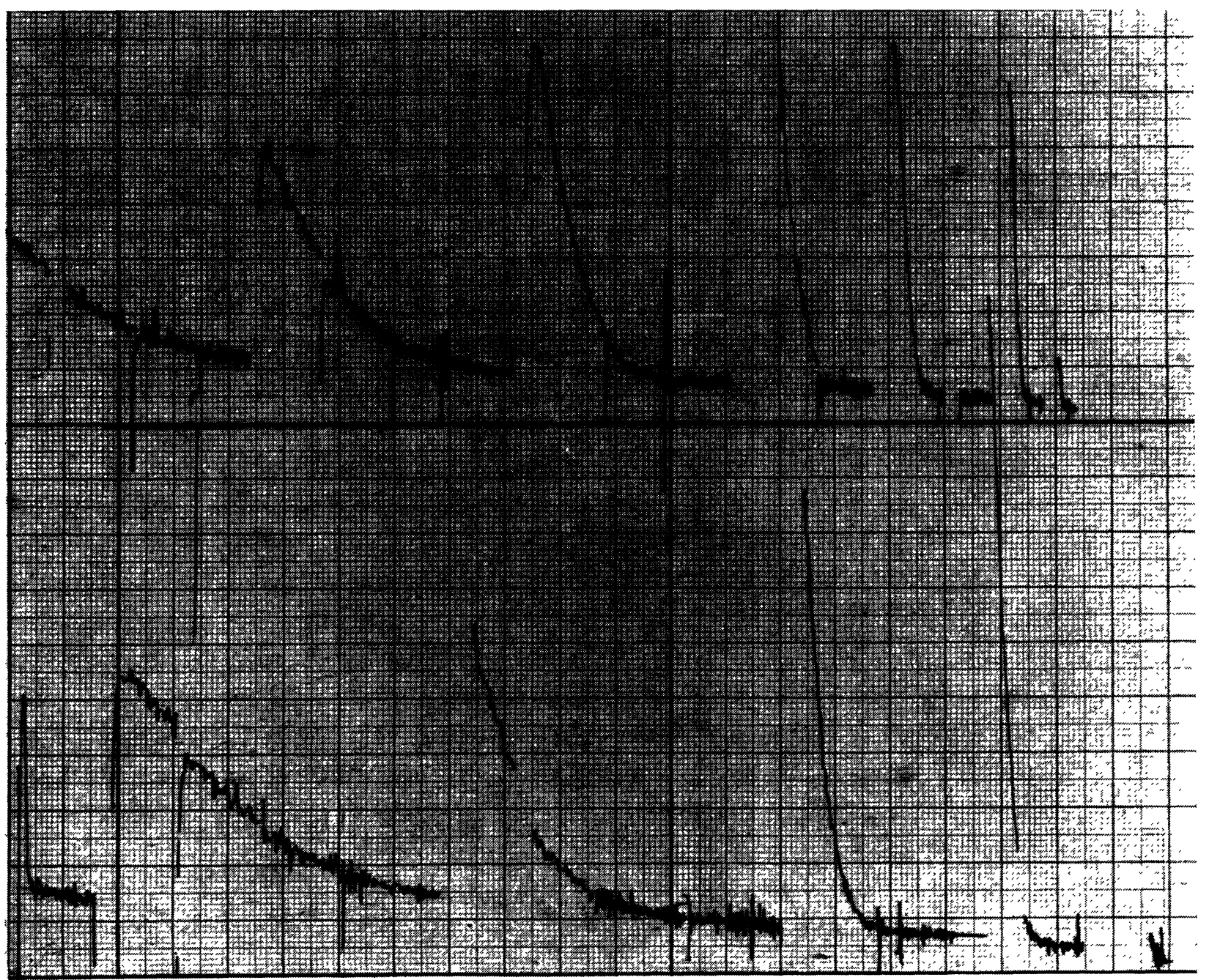

FIG. 4. - The recording of $\dot{M}$ (vertical axis) versus $M$ (horizontal axis) made on a X-Y recorder for 2 distinct runs during an experiment. A large departure from a linear $\dot{M}(M)$ variation, characteristic of the state of dynamic equilibrium (curve $\mathrm{A}^{\prime}$ of Fig. 3), is observed when a heat pulse is applied. This linear $\dot{M}(M)$ variation is recovered after a transient period the larger the lower the temperature (right side of the figure). 
has been experimentally found sufficient to describe the heat leaks in the range of temperature of interest. By integration we have at "equilibrium »

$$
M=\gamma \mathrm{e}^{-\alpha t}+\beta / \alpha .
$$

The determination of the specific heat follows from figure 3. The coefficients $\alpha$ and $\beta$ are obtained from the graph of $\frac{\mathrm{d} M}{\mathrm{~d} t}(M)$ (curve $\mathrm{A}^{\prime}$ in Fig. $3 b$ ) using the data at equilibrium both preceding and following a heat pulse. Then two values for the integration constant $\gamma$ are determined, one before and the other after a heat pulse by using the data of $M(t)$ at equilibrium (curves $\mathrm{A}$ and $\mathrm{B}$ in Fig. $3 a$ ). The drifts at equilibrium are extrapolated from both sides over the phase of non equilibrium and the temperature difference at any chosen time $t_{0}$ may be calculated. From the above, the adoption of a system for the automatic acquisition of the data which would make the computations more straight-forward seems appropriate.

5. The acquisition of the data. - To perform the specific heat determination according to the method described above, the knowledge of the following data is needed :

$1^{\circ}$ Evolution of $M(t)$;

$2^{\circ}$ Detecting arrival in the equilibrium state;

$3^{\circ}$ Determination of the heating energy supplied to the sample.

The problem of the evolution of $M(t)$ is solved by punching on paper tape the value of $M$ at regular time intervals (every $10 \mathrm{~s}$ in our case). In the mutual inductance bridge (constructed in the laboratory and commercialized by S. E. I. N.) $M$ is opposed by discrete values $M_{\mathrm{b}}$ and the difference between $M$ and $M_{\mathrm{b}}$ is amplified and recorded. The bridge settings (the indications of a commercial A. O. I. P. six decades resistance box) were coded ; a solartron 1604 numerical voltmeter reads the unbalance $V$. A data transfer unit (Schlumberger, DTU) allows punching a "word » composed of an analog part (the indications $V$ of the voltmeter) followed by a digital part (the indication $M_{\mathrm{b}}$ of the bridge).

An intervention of the operator is necessary to change $M_{\mathrm{b}}$ to another value $M_{\mathrm{b}}^{\prime}$ when $V$ becomes too large (approaching the linearity limit of the bridge). In the treatment of the data, small extrapolations from both sides will allow to associating a variation $\Delta V$ of the voltmeter reading with a known variation $\Delta M_{\mathrm{b}}$ in the bridge settings and hence establish the correspondance between $M$ and $V$ :

$$
M=M_{\mathrm{b}}+V\left(\frac{\Delta M_{\mathrm{b}}}{\Delta V}\right)
$$

- The direct detection of the arrival of the equilibrium state on the graph of $\frac{\mathrm{d} M}{\mathrm{~d} t}(M)$ on the $\mathrm{XY}$ recorder (Fig. 4) was left to the operator. A parameter $\mathrm{X}$ which is punched together with the digital part of the "word" has the value 1 in the equilibrium state and is automatically set to 0 (non equilibrium) when the heating is started (time $t_{\mathrm{A}}$ ). It is manually reset to 1 at the time $t_{\mathrm{E}}$, when $\mathrm{d} M / \mathrm{d} t$ becomes again a linear function of $M$ on the XY recorder (Fig. 4). It is the knowledge of the value of this punched parameter $X$ that will define for the computer the state of dynamical equilibrium.

- A second channel of the DTU is used to punch the value of the heating current (measured by the voltmeter on an equivalent circuit) and the time of heating (indicated by a coded Schneider timer) after the end of the heating period and before $t_{\mathrm{E}}$. A third channel is used to punch the values of a resistance thermometer fixed in the sample holder. This enables the calibration against the CMN thermometer of resistance thermometers for other experiments in the laboratory.

Figure 5 shows a block diagram of the data acquisition system. A group of relays transmits the « digital part " of the punched "word " to the DTU; they are triggered by the DTU after the analog part of the "word" has been punched. An external clock triggers the reading of the above channels every $10 \mathrm{~s}$, giving the time basis for the treatment of the data.

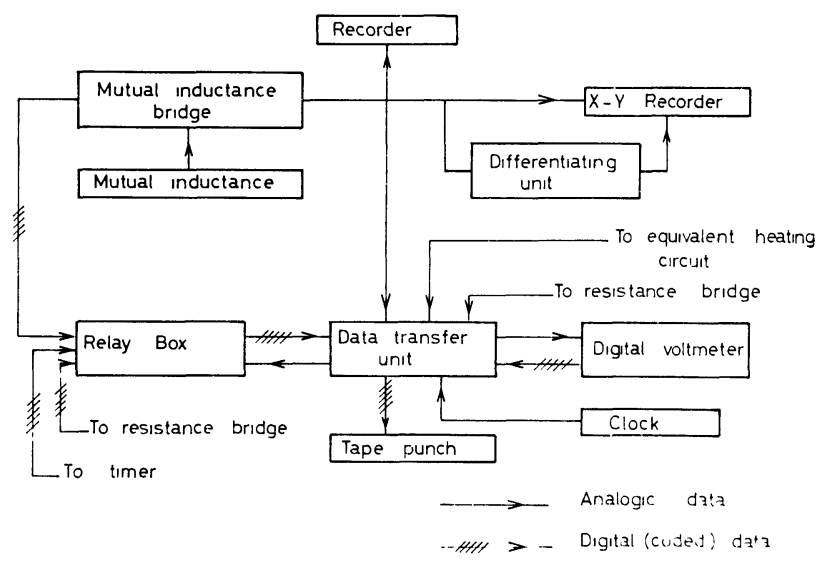

FIG. 5. - Block diagram of the data acquisition system.

6. The treatment of the data. - Figures 6,7 and 8 show the elements of the treatment of the data for one typical run.

- Curve $\mathrm{b}$ in figure 6 shows the reconstruction by the computer of the $\frac{\mathrm{d} M}{\mathrm{~d} t}(M)$ curve also obtained during the experiment by the XY recorder (Fig. 4). - Curve a in figure 6 shows the portions of this $\mathrm{d} M$ $\frac{\mathrm{d} M}{\mathrm{~d} t}(M)$ curve which are retained i. e. the portions at equilibrium $(x=1), \alpha$ and $\beta$, the coefficients of eq. (5), are calculated as explained before by fitting to a straight line (using the least-mean-square pro- 


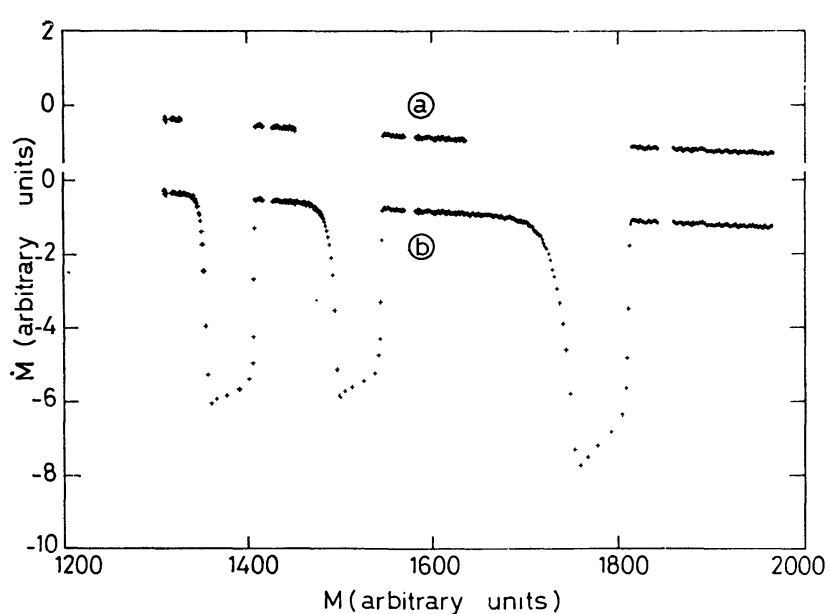

FIG. 6. $-\dot{M}$ versus $M$ as given by the computer from the data punched during the experiment (curve $b$ ). This figure is the restitution of a part of figure 4 . The points that are out of equilibrium are not well described here since $\dot{M}$ has been defined by $\frac{M(t+100)-M(t)}{100}$. Curve $(a)$ shows the part of figure $(b)$ retained by the computer in the extrapolation of the drifts.

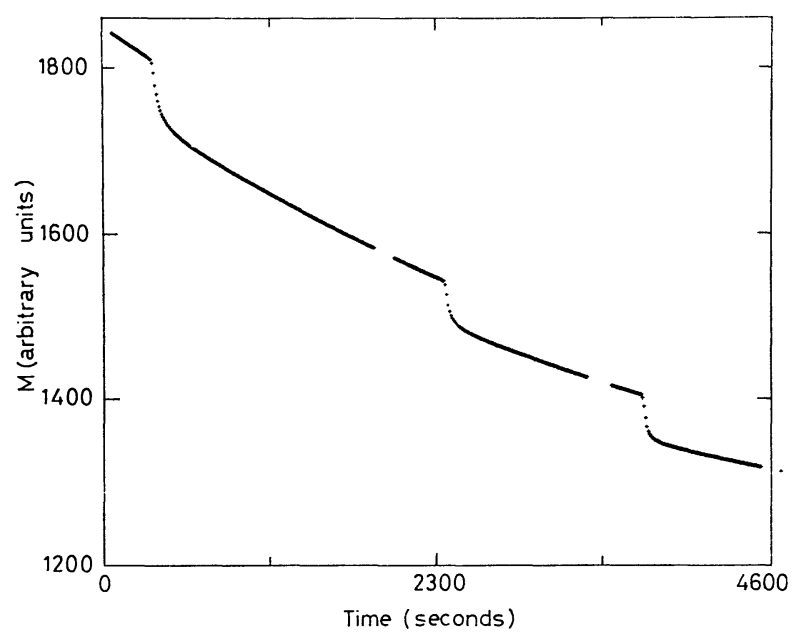

Fig. 7. - A part of a run of $M$ versus time restituted by the computer. The three steps shown correspond to the same three specific heat point as in figure $6(34<T<93 \mathrm{mK})$.

cedure) the two successive portions corresponding to the " equilibrium » data which immediately precede and follow each heating of the sample.

- In figure 7, a part of a typical run $M(T)$ is reconstructed (corresponding to the curve b in Fig. 6). The steps produced by the supplied energy are clearly seen. (The achievement of equilibrium is not so easily detected as in Fig. 4 or 6.) The blank spaces in figure 7 correspond to the few points where an intervention of the operator was necessary to change the bridge settings $(x=2)$ [7]. The values of $\alpha$ and $\beta$ being known, the different values of $\gamma$ (eq. (6)) are calculated using a least-mean-square procedure with the data of the $M(t)$ curve at dynamic equilibrium.

- Figure 8 shows the actual values of $M$ and the extrapolations for a typical determination of a speci-

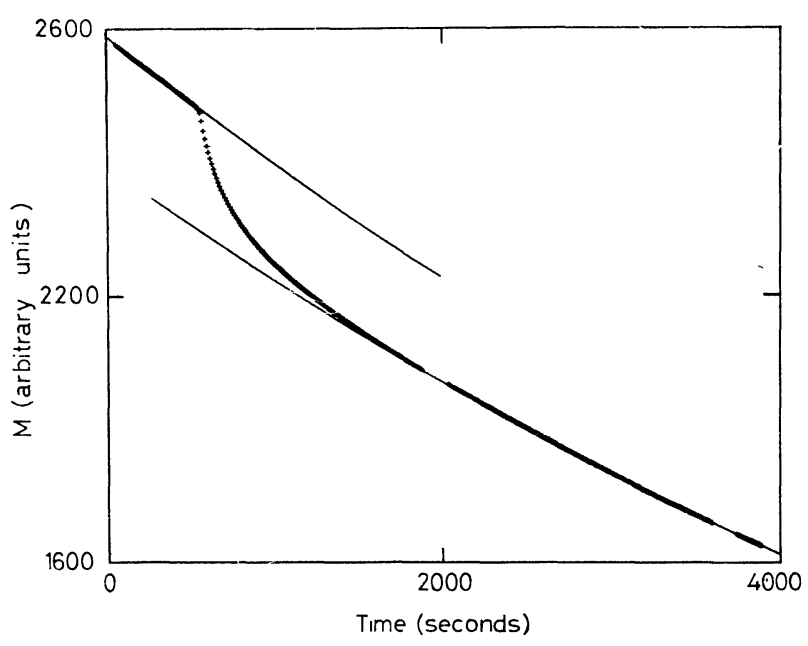

FIG. 8. - The evolution of $M$ versus time during a typical specific heat determination at $23 \mathrm{mK}$ (crosses) and the extrapolations made by the computer (continuous line).

fic heat point. From the values of $M$, the temperature $T$ is recalculated using the calibration law $M=(a / T)+b$ and the specific heat is obtained.

7. Estimation of the errors. - The error on the specific heat is, using the definition $M=(a / T)+b$

$$
\frac{\delta C_{\mathrm{p}}}{C_{\mathrm{p}}}=-\frac{\delta(\Delta T)}{\Delta T}=-\frac{\delta(\Delta M)}{\Delta M}+\frac{2 \delta M}{\delta M}
$$

where $\Delta M$ is the difference between the extrapolated drifts of figure 8, which may be expressed as (eq. (6)) :

$$
\Delta M=\mathrm{e}^{-\alpha t_{0}} \Delta \gamma .
$$

Using (6) and (9) we may write (8) at very low temperatures as :

$$
\frac{\delta C_{\mathbf{p}}}{C_{\mathrm{p}}}=-\alpha \Delta t_{0}+2 \frac{\delta \gamma}{\gamma}-\frac{\delta \Delta \gamma}{\Delta \gamma} .
$$

The term $\alpha \Delta t_{0}$ is connected with the choice of the instant $t_{0}$ at which the estimation of $C_{\mathrm{p}}$ must be performed. The other terms arise from the difference in temperature between the sample and the thermometer, due to the large response time of the latter.

\subsection{UNCERTAINTY DUE TO THE DETERMINATION OF} $t_{0}$. - The largest errors will be present for small heat capacities when $\alpha$ is maximum (from eq. (4) and (5)) and equal to $10^{-4} \mathrm{~s}^{-1}$, and at the lowest temperatures when the response times are long. The choice of $t_{0}$ in the intermediate region between $t_{\mathrm{A}}$ and $t_{\mathrm{E}}$ should be performed by applying the rule of areas on the $M(t)$ diagram : from (4) and (5), $M$ is proportional to $\dot{q}$, so that the heat exchanged with the outside is proportional to the area under the curve $M(t)$ (see ref. [4]). The approach to dynamic equilibrium in $M(t)$ is nearly exponential. The rule of areas then leads to choose $t_{0}$ at $t_{\mathrm{A}}+0.7 \tau$, where $\tau$ is 
the time constant of the exponential. For small heat capacities this time constant is dominated by the response time of the CMN thermometer which is found to be [8]

$$
\tau_{0}=3 T^{-3} \mathrm{~ms}
$$

in qualitative agreement with data reported in reference [9]. At $T=30 \mathrm{mK}$, this leads to $t_{0}=t_{\mathrm{A}}+100 \mathrm{~s}$. In fact, with samples of small heat capacities and rather good diffusivities, the heat input rapidly diffuses through the sample (which is overheated) and then flows to the thermometer which appears underheated as in figure 8. From reference [4], this would lead to a choice of $t_{0}$ closer to $t_{\mathrm{A}}$ than $0.7 \tau$, which appears as a maximum estimation for $t_{0}$.

For practical reasons we have choosen $t_{0}$ at the end of the heating period, which was of the order of $20 \mathrm{~s}$ at the lowest temperatures.

Then, at $T=30 \mathrm{mK}$

$$
\frac{\delta C_{\mathrm{p}}}{C_{\mathrm{p}}}<1 \% \text {. }
$$

7.2 ERror DUE TO THE RESPONSE TIME OF THE THERMOMETER. - At dynamic equilibrium, the temperature of the sample differs from that of the thermometer by a quantity corresponding to

$$
\delta \gamma=\dot{M} \tau_{0}=\tau_{0}(-\alpha M+\beta)
$$

where we have used eq. (5). At low temperatures, with a time constant $\tau_{0}$ proportional to $T^{-3}$ (as quoted previously) we have to first order :

$$
\delta \gamma \sim-\tau_{0} \alpha \gamma \sim-\varepsilon \gamma^{4} .
$$

The error $\delta(\Delta \gamma)$ on the evaluation of $\Delta \gamma$ at $t_{0}$ is :

$$
\delta(\Delta \gamma)=\delta(\gamma+\Delta \gamma)-\delta(\gamma)=\Delta \gamma \frac{\mathrm{d}(\delta \gamma)}{\mathrm{d} \gamma}
$$

Using (11), this gives :

$$
\frac{\delta(\Delta \gamma)}{\Delta \gamma}=-4 \varepsilon \gamma^{3}=-4 \tau_{0} \alpha
$$

Introducing the results of (11) and (12) in eq. (10) we obtain

$$
\frac{\Delta C_{\mathrm{p}}}{C_{\mathrm{p}}}=2 \tau \alpha
$$

For small heat capacities at $T=30 \mathrm{mK}, \Delta C_{\mathrm{p}} / C_{\mathrm{p}} \sim 2 \%$, which is a maximum estimation of this error in all practical cases.

7.3 RANDOM ERRORS. - As shown in figure 7, a typical run from 16 to $70 \mathrm{mK}$ will allow the determination of 4 to 6 values of $C_{\mathrm{p}}(T)$. Then another cycle is started by cooling the sample via the superconducting thermal switch. In a typical experiment 3 to 7 runs are made below $100 \mathrm{mK}$ and the scatter of the experimental points is a good estimation of the random errors. With the method described in this paper, the scatter has been reduced to a few percent for the different runs, as may be seen in the measurements of the Au-Co-Fe [2] and Pt-Co [1] systems. The standard graphical method of linear extrapolation of the drifts leads to a much larger scatter, in the same experimental conditions, as may be seen in the results obtained for the $\mathrm{Au}-\mathrm{Co}$ [2] system. Thanks to this method, the terms in $1 / T^{4}, 1 / T^{6} \ldots$ of the nuclear Schottky anomalies have been observed for the first time in dilute alloys containing $3 \mathrm{~d}$ transition impurities [1].

8. Nuclear specific heat of $\mathrm{Cu}-\mathrm{Mn}$ alloys. - Nuclear orientation measurements yield for the hyperfine field $H_{\text {eff }}$ on the $\mathrm{Mn}$ nuclei in $\mathrm{Cu}-\mathrm{Mn}$ alloys a value of $280 \pm 10 \mathrm{kOe}$ [10], in disagreement with the estimation of $H_{\text {eff }}=430 \mathrm{kOe}$ from previous specific heat data [11]. The presence of a large contribution of the matrix, which could not be separated from the impurity contribution in the specific heat measurement, was advanced as an explanation for an overestimation of $H_{\text {eff }}$.

As a test to the accuracy of the method discussed above we have measured between $20 \mathrm{mK}$ and $100 \mathrm{mK}$ the specific heat of a $\mathrm{Cu}-\mathrm{Mn}$ sample of nominal concentration 1 at. $\%$, with the aim of separating if possible the impurity contribution from the host contribution.

The $14 \mathrm{~g}$ sample $(\varnothing=7 \mathrm{~mm}, 1=20 \mathrm{~mm}$ ) was prepared by the metallurgical group of our laboratory, from $99.999 \%$ Asarco copper $\left(\varnothing=3 / 8^{\prime \prime}\right)$ and $99.99 \%$ Johnson Matthey manganese. The specimen was induction melted by a semilevitation technique on a water cooled base plate and cast in a water cooled copper mold [12]. The actual concentration determined by absorption spectroscopy was 0.901 at. $\% \mathrm{Mn}$.

The nuclear specific heat, $C_{\text {nuclear }}$, is plotted versus temperature in figure 9 (circles). $C_{\text {nuclear }}$ has been obtained from the experimental results by substracting a linear contribution $\gamma T$, with $\gamma=3.7 \mathrm{~mJ} / \mathrm{K}^{2}$. mole, due to the electronic contribution of the host $\left(0.7 \mathrm{~mJ} / \mathrm{K}^{2}\right.$.mole) and to the magnetic ordering contribution of the electronic spins $\left(3 \mathrm{~mJ} / \mathrm{K}^{2}\right.$. mole) [13]. In fact this $\gamma T$ term corresponds to a small correction on the experimental values attaining $5 \%$ at $0.1 \mathrm{~K}$ and completely negligible in the lower temperature range.

The Schottky anomalies expected from the Zeeman splitting of the Mn nuclear levels are also plotted in this figure for 3 different values of the hyperfine field : 280 (curve A), 325 (curve B) and $430 \mathrm{kOe}$ (curve $\mathrm{C}$ ), with the concentration $c=0.9$ at. $\%$. The following conclusion can be drawn :

$1^{\circ}$ For temperatures higher than $50 \mathrm{mK}$ (the range where Du Chatenier and Miedema experiments were performed) the observed specific heat is best described by the high temperature tail of the Schottky 


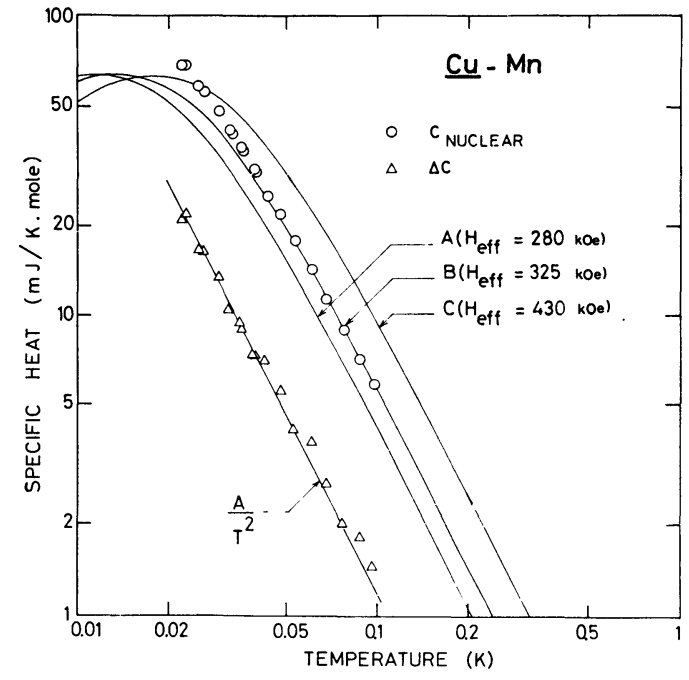

Fig. 9. - The specific heat as a function of temperature in a $\log -\log$ plot for the Cu-Mn sample. The nuclear specific heat (circles) is obtained from the experimental results by substracting the $\gamma T$ contribution. Curves A, B and C are Schottky anomalies corresponding to $H_{\text {eff }}=280,325$ and $430 \mathrm{kOe}$. The excess $\Delta C$ of the nuclear specific heat above curve A follows a $T^{-2}$ law (triangles) and is the contribution attributed to the host nuclei.

anomaly calculated with $H_{\text {eff }}=325 \mathrm{kOe}$ (curve B) and not with $H_{\text {eff }}=430 \mathrm{kOe}$ (curve C), as stated in reference [11].

$2^{\circ}$ Below $50 \mathrm{mK}$ it appears that curve B cannot account for the experimental data and so the experimental points cannot be described by a single Schottky anomaly.

$3^{\circ}$ The experimental values are always higher than curve $\mathrm{A}$, which would correspond to the contribution of $\mathrm{Mn}$ impurities with $H_{\text {eff }}$ as determined by nuclear orientation in a sample containing a few p. p. m. of $\mathrm{Mn}$.

The excess $\Delta C=C_{\text {nuclear }}-C_{\text {curve } \mathrm{A}}$ is plotted in the same figure (triangles), and is well described by an $A T^{-2}$ law in the whole temperature range, with $A=12.0 \mu \mathrm{J} . \mathrm{K} / \mathrm{mole}$. In fact an $A T^{-2}$ law acceptable within experimental error is obtained if $H_{\text {eff }}$ is between 280 and $295 \mathrm{kOe}$, the corresponding values for $A$ being in the range between 12 and $9 \mu \mathrm{J} . \mathrm{K} / \mathrm{mole}$. The permanence of such a $T^{-2}$ law down to $20 \mathrm{mK}$ requires a small value of the nuclear level splitting and thus of $H_{\text {eff }}$ (for large $H_{\text {eff }}$ a departure to the $T^{-2}$ asymptotic behaviour should be observed, as in the case of curves A, B and C). On another hand, the large value of $A$ requires a large number of nuclei probing this small hyperfine field. We are then led to conclude that this contribution comes from the host nuclei.

For 1 mole of Copper :

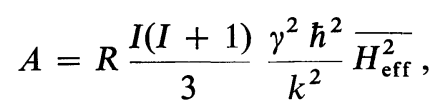

where $I$ and $\gamma$ are respectively the spin and gyromagnetic ratio of the copper nuclei, $H_{\text {eff }}^{2}$ the mean value of the square of the hyperfine field induced on those nuclei, and the other constants have their usual meaning. Let $H_{\text {eff }_{2}}$ be the hyperfine field induced by a manganese atom of the ith shell containing $z_{i}$ sites on one copper nucleus at the origin, the above expression becomes [14] :

$$
A=c(1-c) R \frac{I(I+1)}{3} \frac{\gamma^{2} \hbar^{2}}{k^{2}} \sum_{i} z_{i} H_{\mathrm{eff}_{i}}^{2},
$$

where $c$ is the manganese concentration. The contact hyperfine field $H_{\text {eff }}$ may be expressed as a function of the spin polarization $n\left(r_{i}\right)$ of the conduction band at the site $i$ by [15] :

$$
H_{\text {eff }_{i}}=\frac{\xi}{2 g_{I}} a_{A} \Omega_{0} n\left(r_{i}\right)
$$

where $\Omega_{0}$ is the atomic volume and where we assume for the other parameters in copper the values $[16,17]$

$$
\begin{aligned}
\xi & =\left\langle|\Psi(0)|^{2}\right\rangle_{F} /\left\langle|\Psi(0)|^{2}\right\rangle=0.33 \\
a_{A} & =0.190 \mathrm{~cm}^{-1} \\
g_{I} & =7.39 \times 10^{-24} \mathrm{erg} . \mathrm{G}^{-1} .
\end{aligned}
$$

The asymptotic form of the spin polarization amplitude $n\left(r_{i}\right)$ will be according to Blandin and Friedel [15] :

$$
n\left(r_{i}\right)=-\frac{5}{4 \pi^{2} r_{i}^{3}} \sin \left(\varphi^{+}-\varphi^{-}\right) \cos \left(2 k_{F} r+\varphi^{+}+\varphi^{-}\right)
$$

where $\varphi^{+}$and $\varphi^{-}$are the phase shifts for spin up and spin down directions. Assuming the $\mathrm{Mn}$ ions carry a moment of $4 \mu_{\mathrm{B}}$ :

$$
\begin{aligned}
& \varphi^{+}=0 \\
& \varphi^{-}=\pi / 5 .
\end{aligned}
$$

The summation in (14) converges rather quickly and needs not to be extended further than a few nearest-neighbour shells. Calculations up to the 20th neighbour yield for $A$ the value of $1.5 \mu \mathrm{J}$. K/mole.

In this calculation the contribution of the first four shells is preponderant and the use of the asymptotic expression (16) may be of doubtful validity at such distances. A calculation made by Parlebas [19], taking into account the band structure of $\mathrm{Cu}$ leads for the spin polarisation amplitude on the first neighbour shells to values that are equal or somewhat smaller than those given by (16). On the other hand the calculations of Geldart [20] using the Anderson model for a free electron gas and taking into account the magnetic ion structure yield for the first impurity neighbours values of $n(r)$ which are considerably larger than those given by (16). Using his values up to the 3rd neighbour shell and (16) after that, $A$ is found to be $6 \mu \mathrm{J} . \mathrm{K} / \mathrm{mole}$. The agreement between this experiment and the predictions of the theory appears satisfactory considering the approximations 
involved, especially the questionable validity of (16), where correlations effects have been neglected.

9. Conclusion. - In low temperature calorimetry where the contact resistances added to the bad diffusivity of the CMN introduce large thermal response times, the time derivative of the mutual inductance bridge signal $\dot{M}$ seems to be a very useful parameter. For small-heat-capacity samples, the time constant of the system composed by the sample, the sample holder, and the CMN is given by the CMN time constant which in our case follows the law

$$
\tau_{0}=3 . T^{-3} \mathrm{~ms} \text {, }
$$

in the temperature range between $20 \mathrm{mK}$ and $100 \mathrm{mK}$, attaining thus about 6 minutes at $20 \mathrm{mK}$. On this time scale the temperature drifts are not linear, and the recovery of a state of dynamic equilibrium after a heat pulse is not easily detected in the graph of $M$ vs.time. The recording of $\dot{M}$ versus $M$ made during the experiment enables one to recognize more easily the state of dynamic equilibrium and may be a useful technique in other specific heat measurements at any temperature when problems of bad diffusivity are present.

For the extrapolation of the non-linear temperature drifts a more complex machinery is necessary. The graphical method must be replaced by a computer treatment of the data describing the evolution of $M$ versus time. The observation that for our apparatus $\dot{M}$ or $\dot{q}$ is proportional to $M$ enables us to use a simple analytical law to perform the extrapolation of the drift. The data-acquisition system built using commercially available equipment to which only minor modifications have been made permits straightforward and rapid treatment of the data by a computer.

The above method greatly reduces the systematic and random errors introduced by the linear graphical extrapolation method making possible, for example, the observation of terms of higher order in $T^{-2}$ in the series representing the nuclear Schottky anomaly of dilute alloys containing transition impurities. This allows to separate the impurity contribution from the host contribution to the nuclear specific heat of a $\mathrm{Cu}-\mathrm{Mn}$ alloy. The experimental results are analysed as the sum of a Schottky anomaly due to the impurities, corresponding to the hyperfine field of $280 \mathrm{kOe}$ found by nuclear orientation, and a $T^{-2}$ term due to the impurities, the amplitude of which is found to be in the range predicted by theoretical estimations.

Ackwnowledgments. - We thank the metallurgical group of the laboratory for the preparation of the $\mathrm{Cu}-\mathrm{Mn}$ sample and the data processing group for advice.

\section{References}

[1] Costa-Ribeiro, P., Saint Paul, J. and Thoulouze, D. to be published in J. Low Temp. Phys.

[2] Costa-Ribeiro, P., Souletie, J., Thoulouze, D. and Tournier, R., J. Physique 32 (1971) C 1753.

Costa-Ribeiro, P., Souletie, J. and Thoulouze, D., Phys. Rev. Lett. 24 (1970) 900.

[3] Keyston, J. R. G., Lacaze, A. and Thoulouze, D., Cryogenics 8 (1968) 295.

[4] Keesom, Q. H. and Kox, J. A., Proc. Acad. Sci. Amst. 35 (1932) 294.

Parkinson, D. H. and Quarrington, J. E., Proc. Phys. Soc. A 67 (54) 569.

[5] Miedema, A. R. and Haseda, T., Proceedings of I. I. 2, edited by A. Van Itterbeck (Pergamon Press) London (1961).

[6] Abel, W. R., Anderson, A. C., Black, W. C. and Wheatley, J. C., Physics 1 (1965) 337.

[7] The time response of the electronic not being instantaneous, a wrong value of $M$ may be punched due to this intervention. This is why the operator indicates his intervention by setting to 2 the parameter X. The values of $M$ punched during the change preserve the time basis.

[8] For low temperatures $M \sim a / T$ and so $\tau_{0}$ is also the time constant in the graph of $T$ versus time, exhibiting the same $T^{-3}$ dependance already noted in reference 9 .
[9] Gregers-Hansen, P. E., Krusius, M. and Pickett, G. R., Phys. Rev. Lett. 27 (1970) 38.

[10] Campbell, I. A., Compton, J. P., Williams, I. R. and Wilson, G. V. H., Phys. Rev. Lett. 19 (1967) 1319.

Flouquet, J., Phys. Rev. Lett. 25 (1970) 288.

[11] Duchatenier, F. J., Miedema, A. R., Physica 32 (1966) 403.

[12] Bethoux, O., Ferrari, J., Cornut, B., Rev. Appl. Phys. 5 (1965) 865.

[13] Phillips, N. E., C. R. C. Critical Reviews in Solid State Sciences 2 (1971) 467.

[14] Dreyfus, B. and Thoulouze, D., J. Appl. Phys. 39 (1968) 1320.

[15] Caroli, B. and Blandin, A., J. Phys. Chem. Solids 27 (1966) 503.

[16] Narath, A., Phys. Rev. 165 (1968) 506.

[17] Knight, W. D., Solid State Phys., vol. 2.

Seitz, F. and Turnbull, D. (Academic Press, N. Y.) 1956 Chap. 2.

[18] Blandin, A. et Friedel, J., J. Physique Radium 20 (1959) 160.

[19] Parlebas, J. C., Thesis (unpublished) Université de Strasbourg France (1972).

[20] Geldart, D. J. W., Phys. Lett. 38A (1972) 25. 